\title{
„Blitze, die plötzlich ins Innere der Sachen schießen“. Anmerkungen zum Briefwechsel zwischen Wilhelm von Humboldt und Friedrich Schiller
}

Des éclairs qui, soudain, pénètrent dans l'intérieur des choses ". Notes sur la correspondance échangée par Wilhelm von Humboldt et Friedrich Schiller "Lightning penetrating suddenly the interior of the things". Reflexions on the correspondence of Wilhelm von Humboldt and Friedrich Schiller

\section{Wolfgang Fink}

\section{(2) OpenEdition} Journals

Édition électronique

URL : http://journals.openedition.org/ceg/1037

DOI : $10.4000 /$ ceg. 1037

ISSN : 2605-8359

Éditeur

Presses Universitaires de Provence

Édition imprimée

Date de publication : 30 mai 2016

Pagination : 267-292

ISBN : 979-10-320-0067-0

ISSN : 0751-4239

Référence électronique

Wolfgang Fink, " „Blitze, die plötzlich ins Innere der Sachen schießen“. Anmerkungen zum

Briefwechsel zwischen Wilhelm von Humboldt und Friedrich Schiller », Cahiers d'Études Germaniques [Online], 70 | 2016, Online erschienen am: 17 Dezember 2017, abgerufen am 26 November 2020. URL : http://journals.openedition.org/ceg/1037 ; DOI : https://doi.org/10.4000/ceg.1037 


\title{
„Blitze, die plötzlich ins Innere der Sachen schießen“
}

\author{
Anmerkungen zum Briefwechsel \\ zwischen W. von Humboldt und F. Schiller
}

Wolfgang FINK

Université Lumière Lyon 2

Der Briefwechsel zwischen Humboldt und Schiller umfasst 116 zwischen März 1790 und April 1805 geschriebene Briefe, von denen nur 21 aus Schillers Feder stammen. ${ }^{1}$ Zwei Drittel der Briefe fallen zudem in den Zeitraum von Juli 1795 bis Oktober 1796, begleiten noch vor Humboldts Abreise im Frühjahr 1797 nach Italien bzw. Paris das Ende von Schillers „philosophischer Phase“ und dessen Überlegungen zu den grundsätzlichen Unterschieden zwischen den antiken und modernen Bewusstseinsformen in Über naive und sentimentalische Dichtung. Wie Ernst Osterkamp gezeigt hat, erklärt sich dieses doppelte Ungleichgewicht des Briefwechsels vor allem durch Schillers im Brief vom 26. Oktober 1795 formulierte existentielle Frage, inwiefern er als moderner Dichter „angesichts der konstitutiven Distanz der Moderne zum 'Geiste der griechischen Poesie noch Dichter sein’ könne“2, auf die Humboldt immer wieder durch die Aufwertung der modernen Reflexivität und die Betonung des historischen Abstands zur literarischen Produktion der Alten antwortet. ${ }^{3}$

Zwei weitere Elemente, die die zeitliche Konzentration auf die Jahre 1795 und 1796 sowie das Übergewicht von Humboldts Stellungsnahmen erklären, sind jedoch in Betracht zu ziehen. Schiller formuliert sehr früh eine grundsätzliche Skepsis gegenüber der Möglichkeit, durch das Medium Brief entscheidende intellektuelle und persönliche Erfahrungen zu vermitteln ${ }^{4}$, eine Problematik,

1. Der Briefwechsel zwischen Friedrich Schiller und Wilhelm von Humboldt, Berlin, AufbauVerlag, 1962, Bd. I, S. 266. Der Briefwechsel wird fortan mit der Sigel BW zitiert.

2. Ernst Osterkamp, „Fläche und Tiefe. Wilhelm von Humboldt als Theoretiker von Schillers ,Modernität““, in Walter Hinderer (Hrsg.), Friedrich Schiller und der Weg in die Moderne, Würzburg, Könighausen \&t Neumann, 2006, S. 101-116, hier 103.

3. Osterkamps Studie besitzt den immensen Verdienst, die so weit verbreitete These eines einseitigen Einflusses von Schiller auf Humboldt zu korrigieren, wie sie z. B. Franz-Michael Konrad immer noch weiterträgt. Vgl. ders., Wilhelm von Humboldt, Bern, Haupt, 2010, S. 25-38.

4. Das beweist sein Brief an Goethe vom 26. April 1797, in dem es heißt: „Humboldt ist heute fort, ich sehe ihn mehrere Jahre nicht wieder, und überhaupt lässt sich nicht erwarten, dass wir einander noch einmal so wieder sehen, wie wir uns jetzt verlassen. Das ist also wieder ein Verhältnis, das als beschlossen zu betrachten ist und nicht mehr wieder kommen kann, denn 
der sich Humboldt erst in Paris allmählich bewusst wird, wodurch sich der Ton seiner Briefe ändert. ${ }^{5}$ Die diskursive Dimension seiner Korrespondenz tritt zurück und es dominieren zunehmend in apodiktischer Form formulierte Urteile über das kulturelle Leben der Franzosen im Vergleich zu dem der Deutschen. Und vor allem verbinden sich diese Urteile mit Humboldts Versuch, seine „Grille von der Ähnlichkeit der Griechen und Deutschen ins Licht [zu] setzen. " ${ }^{6}$ Dieser Ansatz schlägt sich in zahlreichen Briefen nieder, die zu einer Bestätigung der in dem zeitgleich veröffentlichten Essay Über Hermann und Dorothea formulierten Thesen werden.

Anders gesagt führt der Aufenthalt in Paris Humboldt zu der endgültigen Gewissheit, dass die politische und kulturelle Entwicklung Frankreichs keinerlei Modellcharakter für die deutschen Staaten besitzt. Die Ablehnung des postrevolutionären Frankreichs verbindet sich bei ihm mit einem generellen Unbehagen an dem französischen Kulturleben, das er durch die unterschiedlichen ,nationalen Charaktere' zu erklären versucht. Aus dieser Konfrontation resultiert eine welthistorische Perspektive, nämlich die Frage nach der kulturellen Zukunft Europas, die für Humboldt bedeutet, welche Nation die Nachfolge der Griechen antreten kann. In diesem Zusammenhang entwickelt er die Perspektive einer von den Deutschen zu leistenden europäischen Kultursynthese auf höherem Niveau, die ihrerseits mit den von Schiller in Deutsche Größe formulierten Thesen konvergiert. Ausgangspunkt ist dabei die Opposition von „Äußerlichkeit“ und „Innerlichkeit“ als Unterscheidung von französischem und deutschem

zwei Jahre, so ungleich verlebt, werden gar viel an uns und also auch zwischen uns verändern.“ Emil Staiger (Hrsg.), Der Briefwechsel zwischen Goethe und Schiller, Frankfurt a.M., Insel, 1977, S. 382 .

5. Humboldt vertraut zunächst der von Gellert erneuerten rhetorischen Tradition derzufolge der Briefwechsel das persönliche Gespräch ersetzen kann. So bedauert er nach seiner Abreise aus Jena zwar die täglichen Gespräche mit Schiller (Brief vom 10. Juli 1795; BW I, S. 65) schreibt dann aber aus Tegel: „Sie sehen, lieber Freund, dass ich unsere Briefe wie unsere Gespräche behandle. Ich schreibe hin, was mir gerade einkommt." (Brief vom 22. September 1795 ; BW I; S. 156). Dank der geringen räumlichen - und damit auch zeitlichen - Entfernung sowie der Integration in ein gemeinsames kulturelles Leben scheint die Kommunikation durch den Brief also möglich zu sein. Dies ändert sich jedoch mit der Abreise nach Frankreich. Schon im Herbst 1798 muss Humboldt feststellen: „Ich habe, seit ich Sie verlassen habe, viele neue Richtungen mehr bekommen, meine alten Ideen haben sich geläutert, bestätigt, enger in einen festeren Kreis zusammengezogen ; wie sehr bedürfte ich bei diesem allen des freundschaftlichen Gesprächs, wie würde ich da in wenigen Tagen dadurch weiter vorrücken als jetzt in ganzen Wochen. [...] Im Gespräch mit Ihnen fände ich vielleicht neue Gänge, die mich unerwartet zum Ziel führten.“ (Brief vom 5. September 1798; BW II, S. 173). Im November 1798 schreibt er dann, dass Briefe aus der Ferne „die Natur des Gesprächs, einer gleichsam freien und spielenden Unternehmung“ verlieren. Vgl. seinen Brief an Körner vom 15. November 1798, in W. von Humboldt, Ansichten über Aesthetik und Literatur. Seine Briefe an Christian Gottfried Körner (1793-1830), hrsg. von F. Jonas, Berlin, Schleiermacher, 1880, S. 97.

6. Humboldt, Brief vom 22. September 1795, BW I, S. 157. Zu den Debatten seit Winckelmann über diese angebliche Geistesverwandtschaft vgl. Manfred Fuhrmann, „Die ,Querelle des Anciens et des Modernes“, der Nationalismus und die deutsche Klassik“, in Bernhard Fabian (Hrsg.), Deutschlands kulturelle Entfaltung. Die Neubestimmung des Menschen, München, Kraus, 1980, S. 49-67. 
,Nationalcharakter und den damit verbundenen jeweils spezifischen kognitiven und ästhetischen Fähigkeiten. ${ }^{7}$

Um diesen Zusammenhang zu veranschaulichen, werde ich zunächst Humboldts Unterscheidung zwischen Alten und Modernen skizzieren (I), dann seine Abgrenzung von Frankreich rekonstruieren (II) und schließlich seine Stilisierung der Deutschen analysieren (III). Bei diesem Vergleich der Kulturen stellen die Griechen die Kontrastfolie dar, von der sich die Modernen abheben, ohne jedoch, wie der Vergleich zwischen Franzosen und Deutschen zeigt, über identische kulturelle Waffen zu verfügen.

\section{Die Alten und die Modernen oder Oberfläche und Tiefe, Außen und Innen}

Humboldts Blick auf die griechische Antike ${ }^{8}$ und insbesondere seine Gegenüberstellung von Alten und Modernen beruht, wie Christian Helmreich jüngst noch einmal betont hat, auf einer differenzierten, an Rousseaus Dialektik orientierten historischen Konstruktion. ${ }^{9}$ Einerseits bedauert Humboldt das zunehmende Übergewicht der Berufsarbeit ${ }^{10}$ als ein Passiv der Moderne ${ }^{11}$, das sich in der Suche nach der Befriedigung immer grösser werdender materieller

7. Humboldt wird die Substantivform des Begriffs erst 1830 in der „Vorerinnerung“ zu der Veröffentlichung seines Briefwechsels mit Schiller benutzen: „Die tiefere und wahrere Richtung im Deutschen liegt in seiner größeren Innerlichkeit, die ihn der Wahrheit der Natur näher erhält, in dem Hange zur Beschäftigung mit Ideen und auf sie bezogenen Empfindungen und in allem, was hieran geknüpft ist. Dadurch unterscheidet er sich von den meisten neuen Nationen und in näherer Bestimmung des Begriffs der Innerlichkeit wieder auch von den Griechen." $B W \mathrm{I}$, S. 17. Zur Zirkulation des Begriffes im Fahrwasser Hegels vgl. Wolfgang Fink, "Innerlichkeit” in Gefahr, Rudolf Eucken und die wirtschaftliche Modernisierung des Kaiserreichs“, in Uwe Puschner / Christina Stange-Fayos / Katja Wimmer (Hrsg.), Laboratorium der Moderne. Ideenzirkulation im Wilhelminischen Kaiserreich, Frankfurt a.M., Peter Lang, 2015, S. 75-92.

8. $\mathrm{Zu}$ Humboldts Verständnis der Antike vgl. immer noch Jean Quillien, G. de Humboldt et la Grèce. Modèle et histoire, Lille, Presses universitaires de Lille, 1983.

9. Christian Helmreich, „L'Histoire dans l'Essai sur les limites de l'activité de l'État de Wilhelm von Humboldt (1792), in Olivier Agard / Françoise Lartillot (Hrsg.), Le libéralisme de Wilhelm von Humboldt, Paris, L'Harmattan, 2015, S. 78-80.

10. Wilhelm von Humboldt, Ideen zu einem Versuch, die Grenzen der Wirksamkeit des Staats zu bestimmen, hier zitiert nach der leicht zugänglichen Ausgabe Stuttgart, Reclam, 1995, S. 18-19; 36-38. Im Gegensatz zu Schiller steht Humboldt der Arbeitsteilung differenzierter gegenüber, wie seine Forderung, der Mensch solle möglichst viele „Verbindungen“ eingehen, um so die moderne Einseitigkeit zu überwinden, belegt. (Ibid., S. 22-23; 43). Andererseits illustriert er die Komplementarität dieser „Verbindungen“ jedoch an dem Musterbeispiel der Verbindung von Mann und Frau, was aus seinem Idealbild Frau resultiert, demzufolge „die Weiber eigentlich dem Ideale der Menschheit näher als der Mann“ sind (ibid., S. 40), aber wiederum einer grundsätzlichen Verdammung der Berufsarbeit gleichkommt. Und dennoch ist Humboldt klarsichtig genug, um die antike Sklaverei als ein „barbarisches Mittel“ zu kritisieren, mit dem „einem Theil der Menschheit durch Aufopferung eines andren die höchste Kraft und Schönheit“ gesichert wurden. (Ibid., S. 38).

11. Vgl. Georg Bollenbeck, Eine Geschichte der Kulturkritik. Von Rousseau bis Günther Anders, München, Beck, 2007, S. 7-22. 
Bedürfnisse niederschlage und dazu führe, dass „die innere Ausbildung nicht selten der äußern Wirksamkeit nachgesetzt" ${ }^{12}$ werde. Andererseits stellt Humboldt in den Bereichen der Religion ${ }^{13}$ und der Kunst ${ }^{14}$ eine eindeutige Überlegenheit der Modernen fest, was für ihn eine generelle Erhöhung der kognitiven Fähigkeiten des Menschen beweist. Diese Befunde verbindet er mit einer nachdrücklichen Aufwertung der modernen Reflexivität und antwortet so auf Schillers Interpretation der Modernisierung der Bewusstseinsformen als einer Verlustgeschichte:

Sie sind, was wir waren; sie sind, was wir wieder werden sollen. Wir waren Natur wie sie, und unsere Kultur soll uns, auf dem Weg der Vernunft und der Freiheit, zur Natur zurückführen. Sie sind also zugleich Darstellung unserer verlorenen Kindheit, die uns ewig das Teuerste bleibt; daher sie uns mit einer gewissen Wehmut erfüllen. Zugleich sind die Darstellungen unserer höchsten Vollendung im Ideale, daher sie uns in eine erhabene Rührung versetzen. ${ }^{15}$

Humboldt berichtigt diese nostalgische Perspektive dadurch, dass er die laut Schiller für die Alten spezifische Erfahrung äußerer Harmonie als einen Anthropomorphismus ${ }^{16}$ deutlich macht:

[...] so befindet sich der naive Dichter in dem Zustande, in welchem wir noch nicht die beschränkte Wirklichkeit von dem unendlichen Ideal durch Reflexion zu trennen gelernt haben, in welchem die Menschheit in uns noch ein harmonisches Ganzes ausmacht und wir daher eben diese Harmonie auch in der Natur zu sehen vermeinen. ${ }^{17}$

Humboldt fragt sich nicht, inwiefern die von ihm postulierte „innere Harmonie“ der Griechen, also das „Ideal des harmonischen Menschen“ (Lukács), selber eine Projektion der Klassiker auf die Antike darstellt. Ihm geht es allein darum, die Überlegenheit der modernen Bewusstseinsformen deutlich zu machen:

Wir haben mehr als sie, und es ist möglich, dieses Mehrere poetisch darzustellen. Sie waren bloss, was sie waren. Wir wissen auch, was wir sind, und blicken darüber hinaus. Wir haben durch die Reflexion einen doppelten Menschen aus uns gemacht. ${ }^{18}$

12. Wilhelm von Humboldt, „Das 18. Jahrhundert“, in ders., Werke in fünf Bänden, hrsg. von Andreas Flitner /Klaus Giel, Darmstadt, Wissenschaftliche Buchgesellschaft, 2010, Bd. I, S. 390.

13. Humboldt, Ideen zu einem Versuch, S. 78-81.

14. Laut Humboldt gilt es, die qualitativen Unterschiede zwischen Alten und Modernen anzuerkennen: sobald man von literarischen Gattungen und Epochen spreche, könne er „die naive für nichts andres als eine frühere Stufe und nur die sentimentalische für den Gipfel erkennen." Humboldt, Brief vom 18. Dezember 1795; BW I, S. 268.

15. Friedrich Schiller, „Über naive und sentimentalische Dichtung“, in ders., Werke in fünf Bänden, Berlin / Weimar, Aufbau-Verlag, 1984, Bd. I, S. 249-250.

16. Gleiches gilt in Humboldts Augen für die Religion; vgl. ders., Ideen zu einem Versuch, S. 80-81.

17. Humboldt, Brief vom 18. Dezember 1795; BW I, S. 266.

18. Humboldt, Brief vom 30. April 1803, BW II, S. 234. Vgl. auch folgende Reflexion zu Genuss und Gefühl: „Wir sind einmal ein reflektierendes und sentimentales Geschlecht, und wer unter uns nicht reflektiert, geniesst darum nicht unbefangener; wir beschäftigen einmal die Sinne minder als den Verstand, das Gefühl mehr als die Einbildungskraft; wir brauchen, um auf unsre Weise gerührt zu werden, einen durch Verstand und Gefühl mannigfaltiger ausgearbeiteten Stoff.“ Humboldt, Brief vom 22. Oktober 1803; BW II, S. 261. 
Damit stellt sich die Frage, wie dieser Überschuss an Reflexion und Reflexivität in die Dichtung integriert werden kann, ohne dass „die reine Genialität, der echte Dichtergeist" ${ }^{19}$ der Alten verloren gehen, ein Problem, das sich für Humboldt am Beispiel des Epos‘ konkretisiert:

Wo aber findet er [der Dichter] nun in der neuern Geschichte eine eigentlich epische Handlung, eine solche, in welcher der Mensch allein und unmittelbar handelnd und zugleich als Held auftritt? ${ }^{20}$

Die Frage nach der Möglichkeit „unmittelbarer“ individueller Handlungen in der modernen Geschichte verweist vor dem Hintergrund der Französischen Revolution auf den dem (politischen) Menschen verbleibenden Handlungsspielraum. In dem Zeitalter der „Masse“ ${ }^{21}$ ist auch der Held nur noch eine „Puppe“, während seine Handlungen den Status von banalen „Begebenheiten“ ${ }^{22}$ annehmen. Humboldt versteht diese Entwicklung sicherlich als eine „Regression“ “23, aber dennoch ist sein Blick nicht durchweg pessimistisch, wie ein Vergleich mit Blanckenburg beweist. Dieser hatte zwanzig Jahre zuvor ebenfalls bedauert, dass Heldenfiguren in der Moderne nicht mehr für kollektive Identitäten einstehen und den Gang der Polis souverän bestimmen können. Er hatte daraus die Konsequenz gezogen, der Roman als bürgerlich-moderne Schrumpfform des Epos solle allein „das Innre des Menschen“ und nicht seine „Thaten und Unternehmungen“" ${ }^{24}$ zeigen. Die Aneinanderreihung solcher Handlungen könne nicht das Interesse des Lesers wecken, da sie „nichts als die Oberfläche, das Äußere der geschehenen Dinge“ ${ }^{25}$ erfasse. Humboldt geht scheinbar in die gleiche Richtung, wenn er schreibt:

Wenn die Alten mehr die Natur in ihrer sinnlichen Pracht und Grösse malen, so legt er [Goethe] mehr das Innre der Menschheit dar. ${ }^{26}$

Tatsächlich jedoch will er das Epos im Gattungssystem behalten, obwohl dessen Glanz „mit dem Sinken der Griechischen Sonne erloschen zu sein“ ${ }^{27}$ scheine. Dieser Eindruck sei jedoch trügerisch, denn es geht allein um „die heroische Epopee“, sie müsse man in der Tat in der Moderne „unter die Zahl der Unmöglichkeiten rechnen.“ ${ }^{28}$ Eine Lösung bestehe jedoch darin, „alle epischen Stoffe immer nur aus dem Privatleben und zwar aus derjenigen Menschklasse zu

19. Humboldt, Brief vom 6. November 1795, BW I, S. 208-209.

20. Wilhelm von Humboldt, Über Hermann und Dorothea, in ders., Werke in fünf Bänden, Bd. II, S. 342 .

21. Humboldt, Ideen zu einem Versuch, S. 30-31.

22. Humboldt, Über Hermann und Dorothea, S. 343.

23. Helmreich, „L'Histoire“, S. 78.

24. Friedrich von Blanckenburg, Versuch über den Roman, Faksimiledruck der Originalausgabe von 1774, Stuttgart, Metzler, 1965, S. 18.

25. Ibid., S. 259.

26. Wilhelm von Humboldt, Über Hermann und Dorothea, S. 223.

27. Ibid., S. 343.

28. Ibid. 
nehmen, die wirklich auch jetzt noch natürlicher, einfacher und antiker lebt.“ ${ }^{29}$ Gerade das beweise Goethe:

Denn der Mangel heroischer Charaktere und Handlungen ist das Einzige, wodurch sich Hermann und Dorothea sichtbar von den übrigen Epopeen unterscheidet. ${ }^{30}$

Humboldt teilt also Blanckenburgs Befund, demzufolge der Verlust souveränen politischen Handelns durch die Eroberung einer psychologischen Dimension kompensiert wird, die es nun ästhetisch fruchtbar zu machen gilt. ${ }^{31}$ Gerade deshalb sieht er keinen radikalen Bruch zwischen Antike und Moderne, sondern fordert die Weiterführung der antiken Tradition mit den Mitteln der Moderne. ${ }^{32}$ Sie muss den Bezug des modernen Menschen zu der ihn umgebenden Welt berücksichtigen; projizierten die Alten ihre eigene innere Harmonie auf die äußere Natur und erfuhren so ihre Existenz als ein harmonisches Ganzes, so sei für die Modernen gerade die Trennung zwischen beiden Sphären konstitutiv:

[...] dass der Unterschied des antiken und modernen Charakters, von dem wir hier reden, vorzüglich darin besteht, dass in diesem letzeren das Feld der Betrachtung und der Empfindung mehr abgesondert bearbeitet wird, wodurch denn natürlich die hierauf gerichteten Kräfte eine höhere und mehr energische Tätigkeit erlangen. Dadurch wird aber zugleich der innere Mensch von der äusseren Wirklichkeit getrennt, es wird zwischen beiden eine Grenze gezogen, so dass es nun auch jenseits derselben ein eignes und neues Gebiet gibt. $^{33}$

Da, wo Blanckenburg vom Romancier einen sukzessiv entwickelten Kausalzusammenhang von äußeren Ereignissen und persönlichen Reaktionen fordert, um so die Genese eines individuellen Charakters überzeugend $\mathrm{zu}$ vermitteln und die Einheit von Romanfigur und ,Realität doch noch zu bewahren ${ }^{34}$, insistiert Humboldt auf der Trennung zwischen Individuum und Welt und die psychologisch- reflexive Dimension wird ihm zu einem Widerstandspotential der Wirklichkeit gegenüber, das geschichtsträchtige Dimensionen annimmt.

Einbindung in die äußere Natur und deren Nachahmung bei den Alten, bewusste Absonderung und Dominanz der inneren Natur bei den Modernen, dies wäre eine erste Dichotomie, deren Schematismus Humboldt jedoch sofort relativiert, wie seine Unterscheidung zwischen Schillers und Goethes Vorgehensweisen belegt:

29. Id.

30. Ibid., S. 289.

31. Zu den Aporien von Blanckenburgs Ansatz vgl. Wolfgang Fink, Le peuple, la populace et le prolétariat, Paris, éditions de la MSH, 2002, S. 59-63.

32. Laut Humboldt beruht der Fortschritt der Menschheit „von Stufe zu Stufe der Kultur“ u. a. auf der Weiterwirkung der antiken Ideenwelt. Humboldt, „Das achtzehnte Jahrhundert“, S. 384. Zu seinem kumulativem Fortschrittsverständnis im Sinne Lessings, vgl. ders. Theorie der Bildung des Menschen [Brief an Körner], in ders., Werke in fünf Bänden, Bd. I, S. 236 ff.

33. Humboldt, Über Hermann und Dorothea, S. 225.

34. Blanckenburg, Versuch über den Roman, S. 262. 
Er [Goethe] wirkt mehr von außen, Sie mehr von innen auf den Menschen; man kommt auf beiderlei Weise zum Ziel, aber man fühlt bei Ihnen die eigne innre Kraft höher angestrengt. ${ }^{35}$

Laut Humboldt zeichnet sich Goethes Dichtung durch einen höheren Grad der Nachahmung aus, während Schiller ein größeres Gewicht auf die distanziertreflexive Dimension legt:

Sie sind doch unendlich glücklich, teurer Freund, einen solchen Reichtum in sich zu bewahren, bloss aus sich selbst so viel schöpfen zu können, als genug ist, ein ganzes Leben mit schöner Mannigfaltigkeit auszustatten. [...] Wenn ich bedenke, [...] wie wenig Sie eigentlich in jedem Verstande von außen nehmen, so erfüllt es mich immer aufs neue mit Bewunderung. ${ }^{36}$

Beide konvergieren jedoch laut Humboldt in der Beibehaltung eines prinzipiellen Abstands zur historischen wie zur dargestellten Wirklichkeit, wodurch allein das kritisch-philosophische Potential der Dichtung erhalten bleibt: der „sentimentalische Dichter unterscheidet sich durch die Absonderung des Ideals von der Wirklichkeit.“" ${ }^{37}$ Die Tragweite dieser Reflexion wird deutlich, wenn man bedenkt, dass für Humboldt die Dichtung das Reflexionsmedium „der für den Menschen konstitutiven Verbindung von Gedanke und Empfindung““38 darstellt. Nur so kann sie mit der notwendigen „Neuschaffung der Wirklichkeit von Innen heraus“ verbunden werden, die „den konstitutiven Mangel der Realität durch die Notwendigkeit des Ideals auszugleichen vermag“. ${ }^{39}$ Von diesen Thesen ausgehend entwickelt Humboldt „eine Topologie der künstlerischen Kreativität“, nämlich „die Opposition von Tiefe und Fläche“ ${ }^{40}$, wobei die „Tiefe“, verbunden mit dem scheinbaren Rückzug ins „Innere“, in Opposition zum „Äußeren“ tritt. Schiller ist der moderne Dichter der Tiefe, „weil es für den Dichter-Philosophen keine Nachahmung der Natur, die immer auch eine Nachahmung von Flächen - Oberflächen - ist, geben kann“. ${ }^{41}$ Auf dem Spiel steht die Fähigkeit zur philosophischen Erkenntnis ${ }^{42}$, denn Humboldt folgt der von Foucault analysierten Überwindung des Repräsentationsschemas durch das moderne Wissen, welches die „Tiefe“ an die Stelle der „Oberfläche“ setzt:

35. Humboldt, Brief an Schiller Anfang September 1800, BW II, S. 204. Die Formulierung ist durchaus ambivalent und Schiller hat dieses ,Kompliment ${ }^{*}$ auch entsprechend negativ aufgenommen. Vgl. Günter Oesterle, „Dialog und versteckte Kritik oder 'Ideentausch' und 'Palinodie': Wilhelm von Humboldt und Friedrich Schiller", in Athenäum. Jahrbuch der Friedrich Schlegel-Gesellschaft (17), 2007, S. 135-158.

36. Humboldt, Brief vom 5. Oktober 1795; BW I, S. 168.

37. Humboldt, Brief vom 18. Dezember 1795; BW I, S. 267.

38. Osterkamp, „Fläche und Tiefe“, S. 11.

39. Id.

40. Ibid., S. 12 .

41. Id.

42. Deshalb scheint es auch unangemessen, bzw. schon von der Romantik her gedacht, wenn Vera Bachmann schreibt, „Tiefe” bezeichne im Briefwechsel zwischen Humboldt und Schiller „einen Bedeutungsüberschuss im Sinne einer Unendlichkeit des Dargestellten, welcher sich nicht auf einen Begriff bringen lasse." Vera Bachmann, Stille Wasser. Tiefe Texte ? Zur Ästhetik der Oberfläche in der Literatur des 19. Jahrhunderts, Bielefeld, transcript, 2013, S. 11. Vgl. auch Burkhard Meyer-Sickendieck, Tiefe. Über die Faszination des Grübelns, München, Fink, 2010, der den großen Bogen von der Romantik bis zu Max Scheler schlägt. 
Pour rejoindre le point où se nouent les formes visibles des êtres [...], il faut se diriger vers ce sommet, vers cette pointe nécessaire mais jamais accessible qui s'enfonce, hors de notre regard, vers le cœur même des choses. ${ }^{43}$

Keine Reproduktion der ,Wirklichkeit also, sondern Schaffung einer neuen ästhetischen Realität, die dieser jedoch nicht einfach entgegengesetzt wird, sondern an der sich der Autor wiederum abarbeiten soll:

Um es daher in seiner ganzen Allgemeinheit auszudrücken, muss ich es lieber gleichsam einen Überschuss von Selbsttätigkeit nennen; eine solche, die sich auch den Stoff, den sie bloss empfangen sollte, noch selbst schafft, aber sich hernach mit ihm wie mit einem bloss gegebnen verbindet; die der Materie und der Data nach fast bloss alleinwirkend, aber der Form und dem Schein nach (worauf das Wesen der genialischen Produktion beruht) nur durch Wechselwirkung tätig ist. ${ }^{44}$

In diesem Sinne bedeute die Literatur der Alten die Vorherrschaft der „Empfänglichkeit” und die der Modernen die Dominanz der „Selbsttätigkeit”45, einer Selbsttätigkeit jedoch, die nicht ins Willkürliche abgleiten darf und der daher durch die geforderte „Wechselwirkung” mit dem Stoff enge Grenzen gesetzt sind. ${ }^{46}$ Nur so ist laut Humboldt über den Weg der philosophischästhetischen Erkenntnis der Widerstand dem Gegebenen gegenüber möglich. Ziel ist es, „in die Tiefe sehen und in die Tiefe wirken“ ${ }^{47}$ zu können. Die Auslösung von an die Empfindung gebundenen Erkenntnisprozessen, die das Bewusstsein des Rezipienten verändern, erscheint somit nicht nur als Spezifikum moderner Literatur, sondern auch als die einzige der Moderne gerecht werdende Form der Dichtung. Die „Verbindung von Ideen mit dem Gefühle“ ${ }^{48}$ in an das „Innere“ des Menschen gerichteten Kunstwerken sei den Alten fremd gewesen. Diese Überzeugung schlägt sich nun in Humboldts Semantik bis zur Redundanz nieder; etwa wenn er Schillers „Tiefe“ ${ }^{49}$ lobt, wenn er betont, dieser habe „mehr Tiefe als Fläche“ ${ }^{50}$ oder wenn er schreibt, Schillers Verse „eröffnen dem Geist auf einmal eine unabsehliche Tiefe“. ${ }^{51}$ Diese Ausrutscher haben jedoch nur anekdotischen Charakter und gleiches gilt für die Tatsache, dass es Humboldt gelungen ist, Schiller von der Überlegenheit der Modernen zu überzeugen. ${ }^{52}$ Wichtiger in

43. Michel Foucault, Les mots et les choses, Paris, Gallimard, 1966, S. 252. Diesen Aspekt hatte Heinz Schlaffer in seinem sonst so beeindruckenden Essay übersehen. Vgl. Heinz Schlaffer, Die kurze Geschichte der deutschen Literatur, München, dtv, 2007, S. 54-112.

44. Humboldt, Brief vom 16. Oktober 1795; BW I, S. 180.

45. Humboldt, Brief vom 6. November 1795; BW I, S. 209.

46. Diesen Umschlag ins Willkürliche wird Hegel am Beispiel der Romantiker unter dem Stichwort „absolute Innerlichkeit“ kategorisch ablehnen. Vgl. Georg Wilhelm Friedrich Hegel, Vorlesungen über die Ästhetik II, WA Bd. 14, Frankfurt a.M., Suhrkamp, 1986, S. 143.

47. Humboldt, Brief an Schiller Anfang September 1800, BW II, S. 196.

48. Humboldt, Brief vom 16.0ktober 1795; BW I, S. 180.

49. Ibid.

50. Ibid.

51. Humboldt, Brief vom 18.8. 1795, BW I, S. 88.

52. Das zeigt Schillers Brief vom 25.Dezember 1795, in dem er seinerseits Homer vorwirft, dieser habe „eine unendliche Fläche, aber keine solche Tiefe. Was sie [die Griechen] an Tiefe haben, ist ein Effekt des Ganzen, nicht des einzelnen [...]." Schiller, Brief an Humboldt vom 25. Dezember 1795; BW I, S. 272. 
unserem Zusammenhang ist das Gewicht, das Humboldt der ästhetischen Vermittlung philosophischer Ideen zuspricht:

Dieses nun, die Menschheit selbst in ihren, zugleich durch ihre innere Kraft und die äußere Bewegung bewirkten Fortschritten, hat unser Dichter unsrer Einbildungskraft darzustellen verstanden. ${ }^{53}$

Hermann und Dorothea erlaubt es also dem Leser, über die historischen Fortschritte der Menschheit zu reflektieren und gleichzeitig durch die Tätigkeit seiner eignen Einbildungskraft Vergangenheit, Gegenwart und Zukunft mit einander $\mathrm{zu}$ verbinden. Noch wichtiger ist allerdings die deutlich werdende Gewichtung von „innerer Kraft“ und „äußerer Bewegung“, denn die Ideen stellen in Humboldts Argumentation den eigentlichen Motor der Geschichte dar. ${ }^{54}$ Nur ,innerlich zeigen sich die echten Quellen, die mächtigsten Triebfedern des Lebens“" 55 und deswegen stellt das „Innere“ bei Humboldt gleich in einem dreifachen Sinne eine geschichtsmächtige Potenz dar: zunächst dient es dem kritischen Abstand zum Zeitgeschehen, der allein eine angemessene philosophische Reflexion ermöglicht. Dann ist es unabdingbare Voraussetzung für eine adäquate ästhetische Vermittlung eben dieser Ideen, die dadurch, dass sie sich einer einfachen Reproduktion der ,Wirklichkeit‘ enthalten, jenen ästhetischphilosophischen Überschuss generieren, der seinerseits neue Erkenntnisprozesse auslöst. Damit stellt sich aber auch die Frage, ob alle Dichter der Moderne zu einer solchen Vorgehensweise fähig sind. In seinem Fragment Das 18. Jahrhundert hatte Humboldt notiert, dass der „Grad der inneren Kraft vorzüglich ein Subjekt vor dem andern auszeichnet." 56 Diese These dient ihm, wie seine Briefe aus Paris belegen, auch zur Differenzierung bzw. Hierarchisierung der zeitgenössischen Kulturen und Nationen.

\section{Frankreich: „Nirgends sieht man Energie, Feuer u. Leben."}

Trotz der räumlichen und zeitlichen Entfernung führte Humboldt in Paris zunächst die gemeinsame Tätigkeit mit Goethe und Schiller fort. ${ }^{57}$ Das beweisen nicht nur die Stellungnahmen zu redaktionellen Fragen, sondern auch die Ausarbeitung seines Essays Über Hermann und Dorothea, sowie, nicht zuletzt, Form und Inhalt seiner Briefe. Sie schließen direkt an die Horen an, wie der systematische Ausschluss des Politischen belegt. Er war für die Zeitschrift programmatisch, denn vor allem Schiller befürchtete „eine Verengung des

53. Humboldt, Über Hermann und Dorothea, S. 304.

54. Humboldt, Ideen zu einem Versuch, S. 194-195.

55. Humboldt, Brief an Schiller Anfang September 1800, BWII, S. 191.

56. Humboldt, „Das 18. Jahrhundert“, S. 437.

57. Zu Humboldts Aufenthalt in Paris vgl. Siegfried A. Kaehler, Wilhelm von Humboldt und der Staat, München/Berlin, R. Oldenburg, 1927, S. 151-174; Michel Espagne, „Humboldt à Paris, lecteur de Goethe", in Revue germanique internationale (12), 1999, S. 195-209; Lothar Gall, Wilhelm von Humboldt. Ein Preuße von Welt, Berlin, Ullstein-Prophyläen, 2011, S. 86-98. 
Bewusstseins und eine Verwahrlosung des Geschmacks.“ ${ }^{58}$ Keine Tagespolitik also, wohl aber geschichtsphilosophische Entwürfe, wie sie im Einladungstext gefordert werden:

\begin{abstract}
Indem sie [die Zeitschrift] sich alle Beziehungen auf den jetzigen Weltlauf und auf die nächsten Erwartungen der Menschheit verbietet, wird sie über die vergangene Welt die Geschichte und über die kommende die Philosophie befragen, wird sie zu dem Ideale veredelter Menschheit [...] einzelne Züge sammeln und an dem stillen Bau bessrer Begriffe, reinerer Grundsätze und edlerer Sitten, von dem zuletzt alle wahre Verbesserung des gesellschaftlichen Zustands abhängt, nach Vermögen geschäftig sein. ${ }^{59}$
\end{abstract}

Dieses Programm wird Humboldt in seiner Korrespondenz trotz Schillers selbstironischer Aufforderung, er solle ihn, der sich „Wanderungen“ in der „poetischen Welt“ hingebe, „mit freundschaftlicher Hand in die Wirklichkeit zurück[führen]"60 in die Praxis umsetzen. Gerade deshalb beschreibt er seinen Aufenthalt in Paris in selbstverleugnerischer Stilisierung als den eines politikfremden Ästheten:

[...] dass ästhetische Gegenstände mich, seit ich hier bin, am anhaltendsten beschäftigen. Sie sind gewiss nicht gerade die, auf welche Paris jetzt zunächst führt, aber wer sich wie ich nicht mit Politik abgibt, wird durch den Mangel alles Geschmacks an echter Philosophie doch dahin zurückgetrieben [...]. ${ }^{61}$

Humboldt trennt das Ästhetische vom Politischen und entwirft das Bild seiner angeblichen, durch die allgemeine Politisierung der Pariser Gesellschaft hervorgerufenen Isolierung, wodurch er untertönig schon eine erste Polarisierung suggeriert: hier das politische Frankreich, dort der an Tagespolitik nicht interessierte deutsche Philosoph. Tatsächlich jedoch pflegte Humboldt, wie seine Materialien belegen, nicht nur ein intensives gesellschaftliches Leben, sondern er verfolgte direkt - durch den regelmäßigen Besuch der Sitzungen des Conseil des Cinq-Cents, des Conseil des Anciens und des Institut de France - und indirekt - durch die Lektüre der Parlamentsberichte, von Rousseau ${ }^{62}$ und den anderen Autoren der französischen Aufklärung sowie von Werken seiner Gesprächspartner - das zeitgenössische politische Leben bis ins kleinste Detail. Er versuchte, das neue Steuersystem zu verstehen, analysierte die Entwicklung der Lebensmittelpreise, spekulierte über die Kaufkraft des Volks vor und nach der Revolution, registrierte die Versuche zu erneuten Aufständen ${ }^{63}$, diskutierte

58. Rüdiger Safranski, Goethe \&t Schiller. Geschichte einer Freundschaft. München, Hanser, 2009, S. 101.

59. Friedrich Schiller, „Einladung zur Mitarbeit an den Horen“, ibid., S. 100.

60. Schiller, Brief vom 21. August 1795; BW I, S. 105.

61. Humboldt, Brief vom 20. Januar 1798; $B W$ II, S. 143. Humboldt verwendet dieselbe Strategie in seiner Korrespondenz mit Goethe, in der es heißt, „[...] um das Politische, wissen Sie, bekümmere ich mich nicht." Goethes Briefwechsel mit W. und A. v. Humboldt, hrsg. von Ludwig Geiger, Berlin, Bondi, 1909, S. 52.

62. Wilhelm von Humboldts Tagebücher, hrsg. von Albert Leitzmann, Erster Band 1788-1798, in Wilhelm von Humboldts gesammelte Schriften, hrsg. von der Königlich Preussischen Akademie der Wissenschaften, Bd. XIV, Berlin, B. Behr's Verlag, 1916, S. 570-620.

63. Ibid., S. 434-438; 487; 507. 
mit Bonaparte ${ }^{64}$ - und notierte seine Eindrücke und Beobachtungen so akribisch, dass man seine Materialien zu Recht als ein intimes Portrait von Staat und Gesellschaft unter dem Directoire bezeichnet hat. ${ }^{65}$

Von all dem kein Wort im Briefwechsel mit Schiller. Durch das Nichtgesagte werden die Briefe zu einer radikalen, oftmals willkürlich erscheinenden Verurteilung des zeitgenössischen Frankreichs. ${ }^{66}$ Denn von den Beobachtungen und Studien geht allein das Portrait des Abbé Sieyès in die Korrespondenz ein, wodurch dessen strategische Bedeutung indirekt betont wird. Dieses wie auch andere Portraits wird durch einen erneuten Verweis auf die angebliche Isolierung eingeleitet:

\begin{abstract}
Ein sonderbares und trauriges Gefühl gibt mir die Einsamkeit, in der ich mich mitten in Paris und seitdem ich Dresden verliess trotz aller mannigfaltigen Bekanntschaften befinde. Über gewisse und gerade die angelegensten Ideen nicht nur mit niemand reden zu können, sondern auch so gewiss zu sein, dass niemand weit und breit ist, der nur irgend Sinn, nur irgend Lust oder Fähigkeit hätte, sie zu verstehen ! Fände ich hier wirklich nur einen in dieser Rücksicht interessanten Menschen, d. h. nur einen selbsttätig philosophischen Kopf, so würde es mir ein grosser Genuss sein, unsre Ideen an einer so fremden Eigentümlichkeit $\mathrm{zu}$ prüfen. ${ }^{67}$
\end{abstract}

DieEinsamkeit istals Stilisierung angeblich weitreichender kulturellerUnterschiede zu verstehen, die Humboldt nun an Hand von Literatur und Philosophie deutlich macht. Das erste Beispiel ist eine Aufführung von Phèdre; Humboldt schreibt in Hinblick auf die Schauspielerin Françoise (Mademoiselle) Raucourt:

[...] dass die Raucourt alles nach außen hin, auf die sinnlichen und äußeren Verhältnisse der Wirklichkeit bezog, da es möglich gewesen wäre, alles ebenso zurück auf sich, auf die sentimentale und innere Stimmung eines idealischen Charakters zu beziehen. ${ }^{68}$

Das Verdikt lässt nicht lange auf sich warten: die Raucourt spielt „mit der Heftigkeit und Wildheit, welche dem innigen, mehr melancholischen Gefühl gänzlich zuwiderläuft." ${ }^{69}$ Unnötig zu betonen, dass hier ein deutscher Zuschauer auf eine französische Theateraufführung reagiert, bzw. Humboldt seine Reaktion so stilisiert, dass angeblich grundlegende nationale Gegensätze deutlich werden, die sowohl in den individuellen Charakteren wie in den Kunstwerken, kurz im jeweiligen ,Geist‘ deutlich werden. Möglich wird diese Dichotomie dadurch, dass Humboldt die in dem Austausch mit Schiller erarbeiteten ästhetischen Kategorien konsequent und normativ auf die Pariser Aufführung anwendet. In dem Sinne stellt seine Kritik schon ein erstes indirektes Urteil über die Fähigkeit

64. Ibid., S. 487.

65. Kaehler, Humboldt und der Staat, S. 155.

66. Diese Verurteilung betrifft allerdings allein die kulturellen Eliten, nicht jedoch das einfache Volk. Vgl. Humboldts Brief an Jacobi vom 26. Oktober 1798 in Briefe von Wilhelm von Humboldt an Friedrich Heinrich Jacobi, hrsg. und erläutert von Albert Leitzmann, Halle, Max Niemeyer, 1892, S. 64. Zu Humboldt und Jacobi vgl. Christina M. Sauter, Wilhelm von Humboldt und die deutsche Aufklärung, Berlin, Duncker \&t Humblot, 1989, S. 269-294.

67. Humboldt, Brief vom 7. Dezember 1797, BW II, S. 140.

68. Humboldt, Brief vom 20. Januar 1798, BW II, S. 145.

69. Ibid. 
der Franzosen dar, eine der Situation der Moderne angemessene Literatur zu produzieren. In die gleiche Richtung zielt seine Darstellung der Debatten mit dem Abbé Sieyès. Eine der Diskussionen führt zu einem Austausch über Metaphysik und verwandelt sich später in ein fünfstündiges „feierliches Kolloquium mit allen Metaphysikern, die es hier gibt“. ${ }^{70}$ Das Resultat ist für den Leser mittlerweile vorhersehbar: „Man verstand sich nicht einmal, geschweige denn, dass man sich bekehrt hätte." ${ }^{71}$ Präziser formuliert ergeben sich folgende Unterschiede:

Sich eigentlich zu verständigen ist unmöglich, und das aus einem sehr einfachen Grunde. Sie [die Franzosen] haben nicht allein keine Ahnung, sondern auch nicht den mindesten Sinn nur für etwas, dass außerhalb der Erscheinungen liegt ; der reine Wille, das eigentliche Gute, das Ich, das reine Selbstbewusstsein, alles dies ist für sie ganz und gar unverständlich. [...] Ihre Vernunft ist nicht unsre, ihr Raum nicht unser Raum, ihre Einbildungskraft nicht die unsrige. $^{72}$

Humboldt benutzt die Argumente und Kategorien, mit denen er Schiller von der Überlegenheit der Modernen zu überzeugen gesucht hatte. In seinen Augen bleiben die französischen Philosophen an der Oberfläche der Phänomene stehen, eine Kritik, die er mit der Metapher des Raums zusammenfasst. Dadurch nimmt er nicht nur die Frage der philosophisch-ästhetischen Topographie wieder auf, sondern bringt sie nun in der vergleichenden Einschätzung zeitgenössischer Kulturen zur Anwendung. Die in dieser Gegenüberstellung postulierten Unterschiede zwischen Deutschen und Franzosen werden zu fundamentalen Differenzen verabsolutiert und bestimmen die jeweilige Rolle der Nationen in der Geschichte. Denn selbst wenn Humboldt in der für ihn bezeichnenden Semantik konzediert, Sieyès sei „offenbar ein tieferer Kopf als alle andern“73, so erscheint ihm doch eine philosophische Auseinandersetzung auf dem Niveau des deutschen Idealismus unmöglich:

Könnte er [Sièyes] sich nach deutscher Art entschließen, für sich, für wenige Leser, für die Nachwelt ein System aufzuführen, so würde er unstreitig etwas Großes liefern. Aber dazu ist er zu sehr Franzose. Die Idee des Publikums um ihn her ist gleich da, und so ist alles Schreiben Teil des öffentlichen Lebens. ${ }^{74}$

Wiederum der implizite Verweis auf den Raum und damit die Opposition von Innen und Außen, denn der Verweis auf das öffentliche Leben beinhaltet die für die Romania charakteristische Wendung nach Außen und damit auf die cité. ${ }^{75}$

70. Humboldt, Brief vom 23. Juni 1798; BW II, S. 153.

71. Ibid., S.154.

72. Ibid.

73. Ibid., S.156.

74. Ibid.

75. Hier wie an anderen Stellen nimmt Humboldt natürlich die nationalen Stereotypen des 18. Jahrhunderts auf, verleiht ihnen aber eine philosophische Dimension. $\mathrm{Zu}$ den Stereotypen vgl. die Arbeiten von Ruth Florack (Hrsg.), Tiefsinnige Deutsche, frivole Franzosen. Nationale Stereotype in deutscher und französischer Literatur, Stuttgart / Weimar, Metzler, 2001; dies. (Hrsg.), Nation als Stereotyp. Fremdwahrnehmung und Identität in deutscher und französischer Literatur, Tübingen, Niemeyer, 2000; dies. (Hrsg.), Bekannte Fremde. Zu Herkunft und Funktion nationaler Stereotype in der Literatur, Tübingen, Niemeyer, 2007. 
Schon ein Jahr zuvor, in dem Fragment Das 18. Jahrhundert, hatte Humboldt die „Heftigkeit“ der Franzosen beklagt, die „mehr nach außen hin zum Handeln, als zum grübelnden Nachdenken, Dichten oder Empfinden neigt." ${ }^{76}$ Gerade dafür wird Sieyès nun zum Musterbeispiel:

[...] er dringt in die Tiefe, aber es sind Blitze, die plötzlich ins Innere der Sachen schiessen, nicht ein langsames, mit ausharrender Anstrengung begleitetes Eindringen. In der Revolution ist er unendlich wichtig gewesen; er hat die hauptsächlichsten Basen der Konstitution, das Repräsentativsystem, die Einheit der Republik und die Teilung des Landes gegründet, und seine Ideen sind ein reicher Vorrat gewesen, aus dem andere mit und ohne seine Absicht geschöpft haben. ${ }^{77}$

Auch hier ein Gemeinplatz aus dem Schatz der ,Nationalcharaktere', der in einen Beweis für psychologisch-philosophische Alterität verwandelt wird: deutsche Langsamkeit vs. französische Schnelligkeit bzw. Brillanz. Nun scheint der Abbé Sieyès - daher seine minimale Geistesverwandtschaft mit den Deutschen - weiter zu gehen als seine Landsleute, denn es gelingt ihm laut Humboldt, „ins Innere der Sachen“ vorzustoßen. Tatsächlich jedoch, und dies ist keine Wortklauberei, dringt Sieyès nicht bis in die Tiefe vor, bleibt also auf halbem Wege stehen. Den Franzosen wird somit die Fähigkeit zu wahrer philosophischer Erkenntnis abgesprochen ${ }^{78}$, was umgekehrt bedeutet, dass Humboldt hier für die Deutschen einen ersten Anspruch auf Erkenntnis - bzw. Deutungshoheit formuliert, der für den Rest seiner Darstellung des französischen Kulturlebens folgenreich sein wird. ${ }^{79}$ Dies beweist u.a. seine Kritik an der Methode der Ideologen, die eine unerwartete Wendung nimmt.

Laut Humboldt verzeichnen in Frankreich nur wenige Wissenschaften und Künste Fortschritte; die Philosophie „schlummert“ wie gesehen, während die Dichtung einige schwache und meist vergebliche Anstrengungen macht, „ihrem längst erreichten Maximum nur wieder irgend nahe zu kommen".80 Anders

76. Humboldt, „Das achtzehnte Jahrhundert“, S. 450.

77. Humboldt, Brief an Schiller vom 23. Juni 1798, BW II, S. 158.

78. Welche Bedeutung diese ,Tatsache' für Humboldt besaß, belegen die Notizen zu seinem „Gespräch mit Alexander über die Franzosen“, die die naturwissenschaftliche Kompetenz des Bruders ins Spiel bringen: „In allen Naturwissenschaften aber haben sie für keine andre als mechanische und atomistische Erklärungsarten Sinn, nirgend aber für eigentliche Kraft und Wirkung. Selbst die chemische Affınität verstehen sie nur mechanisch. Daher sind sie zwar von spiritualistischen Verirrungen frei, gehen aber nirgend bis auf den Grund, und haben nirgend die volle natürliche Ansicht der Dinge.“ Humboldt, Tagebücher, S. 506.

79. Laut Siegfried Kaehler leitet die Konfrontation mit der kulturellen Alterität, so wie sie der Abbé Sieyès verkörpert, das Ende von Humboldts Kosmopolitismus ein (Kaehler, Humboldt und der Staat, S. 167). Angemessener ist es wohl zu sagen, dass hier, am Beispiel der Raucourt wie dem des Abbé, Humboldts Kosmopolitismus, seine Bereitschaft, andere Kulturen zu verstehen, an ihre Grenzen stößt.

80. Humboldt, Brief an Jacobi vom 26. Oktober 1798, S. 61. Einige Seiten weiter wird dann fast beiläufıg der entscheidende Vergleich mit der klassischen deutschen Literatur formuliert, nämlich dass „in dem gesamten Schatz ihrer Literatur [...] kein einziges Denkmal vorhanden ist, aus dem sich ein entschlossenes Streben nach tiefer Philosophie, ächter Poesie, oder erhabener und idealischer Sittlichkeit in vollkommener Reinheit, und ohne allen Zusatz irrdischer Schlacken [...] erweisen ließe.“ Ibid., S. 64. Ohne „Schlacken“, also ohne eine das Ideal unmöglich machende direkte Abbildung der Wirklichkeit. Humboldt folgt hier Schillers Kritik 
gesagt versuchen die französischen Autoren vergeblich, den in Humboldts Augen spätestens zu Beginn des 18. Jahrhunderts begonnenen kulturellen Niedergang ${ }^{81}$ einzudämmen. Doch ist es ihnen unmöglich, die für die Moderne notwendige „Tiefe“ zu entwickeln:

[...] weder dichterisch, noch empfindsam, sind sie überall nur raisonnierend, die bildenden Künste haben nie einen ausserordentlichen und originellen Schwung unter ihnen genommen, ihre dichterischen Werke verrathen zweckmässige Anordnung, genaue Regelmässigkeit, und gesunde Lebensphilosphie, aber nirgends jene Tiefe und jenen Reichtum der Einbildungskraft, welche die Griechen und die Engländer charakterisieren [...]. ${ }^{82}$

DieFranzosen besitzen laut Humboldt nicht einmal eine den Griechen vergleichbare „Tiefe“, also Erkenntnisfähigkeit, während sich der Verweis auf die Engländer als vergiftet erweist, da deren Rolle in der Kulturgeschichte Europas beendet ist ${ }^{83}$, wodurch sich die Frage stellt, welche Nation den künftigen kulturellen Motor Europas und damit der Weltgeschichte darstellen wird. ${ }^{84}$ Dies umso mehr, als dass in Humboldts Augen die gegenwärtige französische Philosophie schwerwiegende Konsequenzen für die in Hinblick auf die Zukunft der Moderne zentrale Frage der Moralität besitzt. Der französische Rationalismus mache den „stillen Bau bessrer Begriffe, reinerer Grundsätze und edlerer Sitten, von dem zuletzt alle wahre Verbesserung des gesellschaftlichen Zustands abhängt“" ${ }^{85}$, unmöglich. Dies beweisen die Arbeiten der Ideologen ${ }^{86}$ und ihr in Humboldts Augen für Frankreich repräsentativer Analyse- und Reflexionsmodus, von dem er sich nicht vorstellen kann, dass er „sich von den Fesseln losmachen kann, die ihn an die Wirklichkeit ketten und ihm allen idealischen Aufflug verwehren“. ${ }^{87}$

am Wilhelm Meister. Vgl. Schillers Brief an Goethe vom 8. Juli 1796 in Briefwechsel (Anm. 4), S. 236-241.

81. Humboldt, Brief an Jacobi vom 26. Oktober 1798, S. 64.

82. Humboldt, „Das 18. Jahrhundert“, S. 449. Vgl. auch den Brief an Jacobi, in dem Humboldt festzustellen glaubt, „dass sie [die Franzosen] in dem, was sie Poesie nennen, nur eine gewisse bestimmte äußere Form fühlen, und in dem wahren und ächten, mehr durch den Gehalt, als durch das, was eigentlich Kunst Form ist, angezogen werden.“ Humboldt, Brief an Jacobi vom 26. Oktober 1798, S. 66.

83. Humboldt betont, dass die dichterische Individualisierung und differenzierte Darstellung von Charakteren zuerst durch Shakespeare und englische Romanautoren eingeleitet worden sei, in der Gegenwart aber vor allem durch die deutsche Literatur fortgeführt werde. „Die französische und italiänische Literatur hat in diesem Fach so gut als nichts geleistet.“ Humboldt, „Das 18. Jahrhundert“, S. 435 bzw. 434.

84. Vgl. dazu den lakonischen Ausschluss Frankreichs aus der europäischen Kulturgeschichte: „Wie die Begebenheiten des Menschengeschlechts jetzt vor uns da liegen, so verdanken wir alles, was wir sind, im Ganzen zwei Völkerstämmen: den Griechischen und den Germanischen. Beide haben der alten und der neuen Zeit das Gepräge ihrer Eigenthümlichkeit sichtbar aufgedrückt.“ Ibid., S. 404.

85. Schiller, „Einladung zur Mitarbeit an den Horen“, S. = 100.

86. Laut Foucault gehörten die Theorien der Ideologen noch zum verschwindenden Repräsentationsschema. Vgl. Foucault, Les mots et les choses, S. 253. Zu den Ideologen vgl. zuletzt Yves Citton / Lise Dumasy (Hrsg.), Le moment idéologique. Littérature et sciences de l'homme, Lyon, ENS Editions, 2013, insbesondere das 5. Kapitel: Dominique Kunz Westerhoff, „L'imagination repensée par les Idéologues ou l'homme-machine entre Lumières et romantisme“.

87. Humboldt, Brief an Goethe vom 10. April 1798. 
Die Franzosen seien zwar mit ihrer Philosophie unzufrieden, blieben aber auf den von Condillac eingeleiteten Wegen, denn für tatsächliche philosophische Analyse fehle ihnen „die nothwendige Strenge und Schärfe“. ${ }^{88}$ „Abstraktion von aller äußern Erfahrung“" ${ }^{89}$ dürfe man daher gar nicht erst erwarten, was über die rein kulturgeschichtliche Einordnung hinausgehende Folgen besitzt:

Bey dieser Philosophie muss natürlich aller Begriff ächter Tugend verschwinden, und sich in einen bloß vernünftigen Eigennutz auflösen. Auch ist es dieser letztere, den die hiesigen Moralisten recht allgemein fühlbar machen möchten. ${ }^{90}$

Anders gesagt handelt es sich um eine rein pragmatische Mischung von Utilitarismus und bien séance. Die Franzosen begnügen sich laut Humboldt damit, „tugendhafte Gewohnheiten“ zu schaffen, wobei ihnen die theoretischen Grundsätze (Kant!) vollkommen gleichgültig seien, Gefühle als Schwärmerei gelten, während die Angst vor Fanatismus und Aberglauben zu „Erbitterung und Hass“ der Religion gegenüber führe, wodurch die Moral eine wesentliche Unterstützung verliere und die Aufklärung in eine „noch ungünstigere Richtung“ gehe. ${ }^{91}$

Im Vergleich zu den Frühschriften ist der Verweis auf die Notwendigkeit der Religion zur Absicherung der Moral bemerkenswert. ${ }^{92}$ In unserem Zusammenhang wichtiger ist jedoch die Begründung der französischen Unfähigkeit, eine in die Zukunft weisende Moralphilosophie zu entwickeln. In seinem langen Brief an Jacobi geht Humboldt näher auf dieses angebliche Phänomen ein und versucht, konjunkturelle und überhistorische Faktoren zu unterscheiden. Was die ersteren betrifft, notiert er:

\begin{abstract}
Größtenteils ist diese Stimmung unstreitig eine Folge der Begebenheiten der letztverflossenen Jahre, und der jetzigen Lage. Die Revolution hat viel zu sehr nur physisch gewirkt; die Schreckens Zeit hat mit ihrem eisern Arme Frankreich in eine starre Betäubung versenkt [...]. Großenteils ist also jene Erscheinung nur zufällig und vorübergehend. Aber zum Theil ist sie auch, glaube ich, tief in dem Charakter der Nation gegründet, und dies muss der Philosoph ausspähen, weil er über die Gegenwart hinausblicken will. ${ }^{93}$
\end{abstract}

Bei genauerer Betrachtung erweisen sich diese Gründe aber nicht nur als konjunkturelle, denn selbst diese physische und durch die Revolutionskriege erklärte Erschlaffung ${ }^{94}$ verbirgt für Humboldt etwas anderes, ein grundlegendes

88. Humboldt, Brief an Jacobi vom 26. Oktober 1798, S. 65.

89. Ibid.

90. Ibid.

91. Ibid., S. 66.

92. Einerseits stellt sie zu den in den Ideen zu einem Versuch entwickelten Thesen eine scheinbare Regression dar; andererseits beweist Humboldts Aufwertung des Idealismus gegen diese in Frankreich zu beobachtende Instrumentalisierung der Religion, dass es ihm doch um Moralität ohne Religiosität geht.

93. Humboldt, Brief an Jacobi vom 26. Oktober 1798, S. 60.

94. „Die Erscheinung, die sich einem jetzt am häufigsten aufdrängt, ist Mattigkeit und Schwäche. Nirgends sieht man Energie, Feuer u. Leben.“ Ibid., S. 61. Vgl. die entsprechenden Aufzeichnungen in ders., Tagebuch, S. 593. Zu Humboldts Energie- Begriff vgl. Jean Quillien, S. 213 ff. 
Übel, „das die Revolution freilich nicht geheilt, aber auch wahrlich nicht hervorgebracht hat; das durch sie nur jetzt klarer ins Auge fällt“"95:

Jede Nation nämlich, wie jeder Mensch überhaupt, braucht, dünkt mich, eine innere Triebfeder, eine lebendige immer rege Kraft, aus der sich seine höhere Thätigkeit, sein eigenthümliches Daseyn entwickeln kann. Ein solches inneres Prinzip des Lebens vermisse ich in dieser Nation; u. gerade weil ich dies wahrhaft heilige Feuer, das allein die Menschheit zugleich läutert und nährt, mehr als irgendwo sonst in der deutschen Nation antreffe, so wächst dadurch, wie ich nicht leugne, meine tiefe Achtung, u. meine innige Anhänglichkeit für sie. ${ }^{96}$

Wie so oft entwickelt Humboldt eine Parallele zwischen Individuum und Nation; bedenkt man weiterhin, dass in seinen Augen ein jeder Mensch seinen individueller Beitrag zum Fortschritt der Menschheit liefern kann - die Handlungen eines jeden Individuums haben Auswirkungen auf „das Ganze der Menschheit“97 - so ist sein Urteil über Frankreich nur noch vernichtender:

Der Mensch rückt nicht wahrhaft weiter fort, wenn er nicht Ideale vor Augen hat, wenn nicht die Idee des Guten, Wahren, und Schönen in andern und höhern Bildern, als die uns täglich im bloss logisch Richtigen, Nützlichen und gefällig Harmonischen begegnen, vor uns stehen. Diese Ideale, der Blick auf sie [...] fehlt den Franzosen. [...]. Man vermisst die tiefe Energie des Geistes, die, durch wahre, aber innere Erfahrung bereichert, nicht bloß Verhältnisse von Begriffen, sondern wahres Daseyn entdeckt [...]. ${ }^{98}$

Der modernen Reflexivität gerecht werdende, über die einfache Reproduktion der ,Wirklichkeit' hinausgehende ästhetisch-philosophische Ideale erweisen sich in Humboldts Augen als der Realität und damit auch der Geschichte übergeordnet. Ihre eminente Rolle bestehe darin, nicht nur dem Menschen die notwendige Orientierung zu vermitteln, sondern auch, die individuellen wie die kollektiven Kräfte zu kanalisieren. Auf dem Spiel stehe der moralische Fortschritt der Menschheit, zu dem Frankreich keinen Beitrag leisten könne, da es keinerlei Sensibilität für die geschichtsbildende Kraft des Idealismus besitze. Humboldts Schlussfolgerung ist eindeutig, nämlich „dass das innere und bessere Leben hier meistenteils zerknickt wird." 99 Die Fähigkeit zu wegweisenden Erkenntnissen sei durch das Gewicht der politischen Ereignisse und deren Reproduktion in Philosophie und Kunst jedoch nicht einfach außer Kraft gesetzt. Tatsächlich sei sie in Frankreich dauerhaft zerstört worden, „weil es [das innere Leben] schon viele Generationen hindurch dieselbe Operation erfahren hat." ${ }^{100}$ Seine Energie lässt nach, es „entglimmt“. ${ }^{101}$ Damit stellen sich nun zwei mit einander verbundene

95. Humboldt, Brief an Jacobi vom 26. Oktober 1798, S. 61.

96. Ibid.

97. Humboldt, „Das achtzehnte Jahrhundert“, S. 383.

98. Humboldt, Brief an Jacobi vom 26. Oktober 1798, S. 60.

99. Ibid., S. 64.

100. Ibid.

101. Ibid. Humboldt hatte schon 1791 die Notwendigkeit von Idealen für menschliche Energie und Tugend in einer vergleichbaren Metaphorik formuliert: „Alles, was wir mit Wärme und Enthusiasmus ergreifen, ist eine Art der Liebe. Wenn nun nicht Ein Ideal mehr die Seele füllt, so ist Kälte, wo ehemals Glut war. Ueberhaupt vermag mit Energie nie der zu wirken, der mit allen Kräften auf Einmal gleichmäßig wirken soll. Mit der Energie aber schwindet jede andre Tugend 
Fragen: einerseits, wie es angesichts dieser psychologischen Dispositionen überhaupt $\mathrm{zu}$ einer dominierende Rolle Frankreichs in der europäischen Geschichte kommen konnte und andererseits, welche Zukunftsperspektiven sich auf Grund des französischen Niedergangs ergeben. Die Antwort auf beide Fragen resultiert selbstverständlich aus der Konfrontation mit dem deutschen ,Charakter':

Der französische Charakter ist mehr Verstand als Geist, mehr außer sich, aufs Leben gerichtete, als eigentlich in sich gekehrte und künstlerisch gestimmte Einbildungskraft, mehr Heftigkeit und Leidenschaft als Empfindung. ${ }^{102}$

Die ,Charaktere' erweisen sich wie erwartet als diametral entgegengesetzt. Hier Gedanken und Empfindung ${ }^{103}$, dort nach außen gerichtete Leidenschaft, die dazu führe, dass

[...] notwendig der Verstand überwiegend seyn muss, aber freilich nicht derjenige, welcher im Denken die Tiefen der Dinge ergründet, und im Handeln nach langsamer Überlegung verfährt, sondern der, welcher schnell auffasst, treffend bemerkt, mit Gewandtheit Mittel auffındet, mit Klugheit sie dem Zweck anpasst, und mit rücksichtsloser Kälte seine Entschlüsse ins Werk richtet. ${ }^{104}$

Verbunden mit der eingangs zitierten These, dass in der Moderne „die innere Ausbildung nicht selten der äußern Wirksamkeit nachgesetzt" 105 werde, ergeben sich nun historische Perspektiven. Halten wir zunächst fest, dass die Gegenüberstellung von französischer ,Schnelligkeit" und deutscher ,Langsamkeit moralisch aufgeladen wird, was vor dem Hintergrund der Französischen Revolution eine distanzierende Erklärung dieser Ereignisse bedeutet. Doch Humboldt geht noch weiter. Denn einerseits bietet er hier einen Schlüssel zum Verständnis der vergangenen politischen und kulturellen Vormachtstellung Frankreichs, wobei sich der französische Vorsprung eher als ein psychologisch zu erklärender deutscher Rückstand erweist. ${ }^{106}$ Andererseits impliziert die Kritik am skrupellosen Handeln auch eine Gegenüberstellung von „inneren“ und „äußeren“ Handlungen im Sinne Platons:

In Wahrheit aber war die Gerechtigkeit, wie sich zeigte, zwar etwas dieser Art, aber nicht an den äußeren Handlungen in bezug auf das, was dem Menschen gehört, sondern an der wahrhaft inneren Tätigkeit in Absicht auf sich selbst und das Seinige indem er nämlich in ihm nichts Fremdes verrichten lässt [...]. ${ }^{107}$

hin. Ohne sie wird der Mensch Maschine. Man bewundert, was er thut; man verachtet, was er ist.“ Wilhelm von Humboldt, „Ideen über Staatsverfassung“, in ders., Werke in fünf Bänden, Bd. I, S. 37.

102. Humboldt, Brief an Goethe vom 10. April 1798.

103. Wilhelm von Humboldt, „Über den französischen Nationalcharakter“, in ders., Gesammelte Schriften, Bd. VII.2, S. 587-592 ; vgl. auch ders., Tagebücher, S. 369.

104. Humboldt, „Das 18. Jahrhundert“, S. 450.

105. Ibid., S. 390.

106. Ibid., S. 499.

107. Platon, Politeia, in ders., Sämtliche Werke, Hamburg, Rowohlt, 1958, Bd. 3, S. 169 (443 d). 
Für Platon wie für Humboldt ist das gerechte Leben „das vorteilhafteste, auch wenn es an Erfolgen in der Welt des Handelns und der Macht gebricht. Der wirklich Weise - der Gerechte und daher Glückliche - schert sich im Grunde gar nicht um die Welt der Macht.“ " ${ }^{108}$ Damit nimmt Humboldts Projekt einer von den Deutschen zu leistenden europäischen Kultursynthese Konturen an, wie auch folgende, dem Gesagten nur scheinbar widersprechende positive Würdigung der „äußeren Handlungen“ deutlich macht:

\begin{abstract}
Wir Deutschen unterscheiden immer ganz bestimmt, zwey gleichsam ganz verschiedene Welten, eine unsichtbare und eine sichtbare, ein inneres und ein äusseres Dasein, und vergessen sehr oft, dass wir, indem wir reden, schreiben, u. handeln, aus dem ersteren heraustreten. Dadurch sind wir dunkel, oft [...] unfein, und beinahe immer formlos. Bey den Franzosen ist es gerade das Gegenteil, sie berechnen alles auf die Wirkung, und dies ist es, was im Großen und Kleinen, ihnen die politischen und gesellschaftlichen Vortheile über ihre Nachbarn gibt. ${ }^{109}$
\end{abstract}

Verbindet man diese Reflexion mit den Thesen über den Charakter der Modernen, so wird deutlich, dass Humboldt hier beide, Moderne und Deutsche, gleichsetzt. Andererseits betont er jedoch in demselben Brief, dass sich die „äußeren Handlungen“ der Franzosen bisher als effizienter erwiesen hätten:

Diese Rücksicht auf den Gebrauch und das äußere Leben hindert vielleicht das tiefe Forschen nach Wahrheit, aber es bewahrt auch vor einer Menge Chimären und erleichtert die Verbreitung des wirklich gefundenen; sie lähmt vielleicht den hohen und idealischen Flug der Empfindung, aber sie bricht auch die Gewalt des rohen Naturtriebs [...]. ${ }^{110}$

Laut Humboldt resultiert Zivilisation also aus auf Wirkungsabsicht bezogenem Handeln, aus der Konzentration auf das „Äußere“, weshalb den Franzosen auf Grund ihres historischen Vorsprungs die anderen Nationen als „roh“111 vorgekommen seien. Humboldt leistet hier nicht nur einen entscheidenden Beitrag zur Entstehung der deutsch- französischen Dichotomie Kultur bzw. Bildung vs. Zivilisation ${ }^{112}$, sondern er gibt zugleich zu verstehen, dass Europa von der Zivilisation wieder zur Kultur zurückfınden muss. In seinen Augen ist der Zeitpunkt eines qualitativen Wandels gekommen: bedeutete die bisherige moderne Geschichte Europas die Vorherrschaft der in der Französischen Revolution kulminierenden „äußeren Handlungen“, so gelte es nun, die „inneren Handlungen“ durchzusetzen, was zugleich die Ablösung des bisher von den Franzosen verbreiteten „wirklichen“, also oberflächlichen Wissens durch eine höhere Sichtweise und „tiefere“ Erkenntnisse impliziert. Frankreich hat ausgespielt:

108. Charles Taylor, Quellen des Selbst. Die Entstehung der neuzeitlichen Identität, Frankfurt a.M., Suhrkamp, 1996, S. 224.

109. Humboldt, Brief an Jacobi vom 26. Oktober 1798, S. 67.

110. Ibid., S. 69.

111. Ibid.

112. Vgl. Georg Bollenbeck, Bildung und Kultur. Glanz und Elend eines deutschen Deutungsmusters, Frankfurt a.M., Suhrkamp, 1996. 
Was ich meine ist eigentlich nur das, dass die Richtung ihrer Kultur, nicht bloß ihrer geistigen Tätigkeit einen gerade vom Ziel abführenden Weg anweist, sondern auch die Quellen selbst verunreinigt, aus welchen sie entspringt [...]. ${ }^{113}$

\section{Die Deutschen oder "diese Ekstase, in die mich meine Deutschheit versetzt!"}

Die kommende Rolle Deutschlands in und für Europa resultiert, so wie sie Humboldt in seinen Briefen und Essays skizziert, vor allem aus den in Paris gemachten Erfahrungen und Lektüren. Sie bedeutet nicht das Ergebnis einer frontalen Konfrontation zweier entgegengesetzter Identitäten, sondern steht am Ende eines kurzen, näher zu bestimmenden Bildungsprozesses. ${ }^{114}$ Schiller lässt sich von Humboldts Stilisierungen täuschen, wenn er von dessen angeblicher „alten Deutschheit“ spricht, die in den Briefen zum Ausdruck komme. ${ }^{115}$ Humboldt betont die progressive Änderung in seiner Haltung Frankreich gegenüber, die immer auch einer Vertiefung seiner deutschen Identität bedeutet. So zum Beispiel in seinem Rückblick auf seine Arbeit an Hermann und Dorothea ${ }^{116}$ oder in folgender Mitteilung an Schiller:

Sie sehen, dass ich meine Deutschheit in Paris nicht ablege. Wirklich fühle ich sie auch vielmehr durch den Kontrast hier wachsen; mögen nur die gütigen Göttern dass es im guten Verstande sei. ${ }^{117}$

113. Humboldt, Brief an Jacobi vom 26. Oktober 1798, S. 63

114. Dabei stellt die Arbeit an Über Hermann und Dorothea eine Übergangsperiode dar. Sie übernimmt die Funktion des Gesprächs bzw. der Korrespondenz mit Schiller und bildet damit zugleich einen permanenten Kontrast zum gesellschaftlichen Leben in Paris: „[...], aber weil mir die Stunden, wo sie mich am angelengtlichsten beschäftigte, immer wie eine Zeit vorkamen, die ich an Ihrer Seite, in Ihrem Gespräch verlebte, weil sie mir mitten in dem fremdartigen Zirkel, der mich umgibt und der mich nur darum anzieht, weil er mir fremd ist, jenes bessere und meinen innersten Neigungen angemessenere Dasein versinnlichten, weil mich selbst das Nachdenken über diese Gegenstände mein günstiges Geschick segnen lehrte, in unsrer Heimat geboren zu sein, zugleich mit Ihnen und Goethe zu leben und Sie und Ihre Liebe zu besitzen. Lachen Sie nicht, mein teurer Freund, über diese Ekstase, in die mich meine Deutschheit versetzt!“ Humboldt, Brief vom 19. April 1798, BW II, S. 149-150.

115. „Unser Freund Humboldt, von dem ich Ihnen einen längeren Brief beilege, bleibt mitten in dem neugeschaffenen Paris seiner alten Deutschheit getreu, und scheint nichts als die äußere Umgebung verändert $\mathrm{zu}$ haben. Es ist mit einer gewissen Art $\mathrm{zu}$ philosophieren und $\mathrm{zu}$ empfinden wie mit einer gewissen Religion; sie schneidet ab von außen und isoliert, indem sie von innen die Innigkeit vermehrt." Schiller an Goethe am 29. Dezember 1797 (Briefwechsel, S. 528). Schiller bezieht sich wahrscheinlich auf Humboldts Brief vom 7. Dezember 1797, in dem es heißt: „In der Tat rechne ich es zu den Vorzügen meines hiesigen Aufenthalts, dass mir die deutsche Natur in ihrem Adel und ihrer Vortrefflichkeit erst hier recht klar werden wird.“ $B$ II, S. 141.

116. Humboldt, Brief vom 5. September 1798, BW II, S. 174.

117. Humboldt, Brief vom 20. Januar 1798, BW II, S. 147. Vgl. auch den Brief an Jacobi: „[...] ich habe mich viel $u$. genau um Frankreich $u$. seine jetzige Lage bekümmert; aber ich bin darum schlechterdings nicht aus meiner eigenthümlichen Assiette herausgekommen; ich bin vielmehr mitten in Frankreich nur ein noch eingefleischterer Deutscher als vorher geworden. " Humboldt, Brief an Jacobi vom 26. Oktober 1798, S. 60. 
Diese Behauptung ist für Humboldts Argumentation wichtig, da sie die Verbindung seines persönlichen Bildungserlebnisses in Paris mit den zusammen mit Schiller entwickelten ästhetischen Prinzipien des Idealismus und, nicht zuletzt, den Thesen über die kulturelle Rolle Deutschlands vorbereitet:

\begin{abstract}
Beschränken sich indes alle diese Forderungen nur auf das innere Wesen des Menschen, so dringt ihn doch seine Natur beständig von sich aus zu den Gegenständen ausser ihm überzugehen, und hier kommt es nun darauf an, dass er in dieser Entfremdung nicht sich selbst verliere, sondern vielmehr von allem, was er außer sich vornimmt, immer das erhellende Licht und die wohltätige Wärme in sein Innres zurückstrahle. ${ }^{118}$
\end{abstract}

Die Forderung, zum Fortschritt von „Bildung, Weisheit und Tugend“119 beizutragen richtet sich zwar an das Individuum und ist an die allmähliche Entfaltung von dessen Individualität gebunden. Trotzdem sieht Humboldt im Austausch zwischen dem „Inneren“ und der Außenwelt eine „Entfremdung“, die den Menschen in seiner Identität bedroht. Dies bedeutet zunächst, dass die intellektuelle Verarbeitung äußerer Eindrücke strukturell - wenn auch in umgekehrter Richtung - der Tätigkeit bei der Schaffung idealistischer Kunstwerke entspricht. So, wie die Subjektivität die ästhetische Realität nicht willkürlich gestalten darf, ist umgekehrt der Einfluss der Wirklichkeit auf das Bewusstsein des Individuums in Grenzen zu halten. Anders gesagt erweist sich die von Humboldt immer wieder beschworene „Wechselwirkung“ der „Empfänglichkeit“ des Menschen „mit seiner Selbsttätigkeit“ ${ }^{120}$ als der Entelechie ${ }^{121}$ untergeordnet: sie ist nur Mittel zum Zweck. Sie darf allein der Entfaltung der in dem Individuum bereits angelegten Fähigkeiten und Eigenschaften dienen ${ }^{122}$, was seinerseits auf die vom Autor nachdrücklich verteidigte Theorie der Nationalcharaktere ${ }^{123}$ verweist. Denn selbst wenn Humboldt in dieser Hinsicht versucht, „die Handlungen der Einzelnen“ vom „Schicksale der Masse“124 zu unterscheiden,

118. Humboldt, „Theorie der Bildung des Menschen“, S. 237.

119. Ibid., S. 236.

120. Ibid., S. 237.

121. Vgl. Jürgen Kost, Wilhelm von Humboldt. Weimarer Klassik. Bürgerliches Bewusstsein, Würzburg, Könighausen \& Neumann, 2004. Trotzdem scheint es übertrieben, zu sagen, Individualität sei bei Humboldt „im Grunde genommen unabhängig von äußeren Einflüssen.“ (Ibid., S. 130)

122. Vgl. seine Selbstbeobachtung in einem Brief an Schiller: „Ich habe, seit ich Sie verlassen habe, viele neue Richtungen mehr bekommen, meine alten Ideen haben sich geläutert, bestätigt, enger in einen festeren Kreis zusammengezogen [...]. “ Humboldt, Brief vom 5. September 1798; BW II, S. 173.

123. „Große Massen, Stämme und Nationen, behalten Jahrhunderte hindurch einen gemeinsamen Charakter, und selbst, wo derselbe große Veränderungen erleidet, sind noch die Spuren seines Ursprungs sichtbar. Gleiche Ursachen bringen durch alle Zeiten hindurch gleiche Wirkungen hervor, und durchaus wird man daher im Ganzen ziemlich dieselben Resultate ähnlicher Kräfte finden, denselben Einfluss der äußeren Lagen, dasselbe Spiel der Leidenschaften, dieselbe Macht des Guten und Wahren, mit dem es aus dem verworrensten Gewebe von Begebenheiten und in den mannigfaltigsten Gestalten hervorgeht.“ Wilhelm von Humboldt, „Plan zu einer vergleichenden Anthropologie“, in ders., Werke in fünf Bänden, Bd. I, S. 352. In Paris versucht er durch eine vorsichtige Kritik an Montesquieu, diesen Schematismus zu relativieren. Vgl. Humboldt, Tagebücher, S. 596.

124. Humboldt, „Plan zu einer vergleichenden Anthropologie“, S. 352. 
also ,Nationalcharakter und ,großes Individuum“ voneinander zu trennen, beweist sein Portrait des Abbé Sieyès genau das Gegenteil. Die Orientierung an den ,nationalen' Charakteren ermöglicht gleichermaßen seine Präsentation von Hermann und Dorothea als deutschem Epos von universeller Bedeutung:

\begin{abstract}
All jene Dinge, zeigt uns der Dichter, sind vereinbar durch die Beibehaltung und Ausbildung unseres natürlichen und individuellen Charakters, dadurch dass man seinen geraden und gesunden Sinn mit festem Mut gegen alle äußeren Stürme behauptet, ihn jedem höheren und besseren Eindruck offen hält, aber jedem Geist der Verwirrung und Unruhe mit Macht widersteht. Alsdann bewahrt das Menschengeschlecht seine reine Natur, aber bildet sie aus; alsdann folgt jeder seiner Eigenthümlichkeit, aber aus der allgemeinen Verschiedenheit geht Einheit im Ganzen hervor; alsdann erhalten die äußern Ereignisse und Zerrüttungen die Thätigkeit der Kräfte rege, aber der Mensch formt darum nicht weniger die Welt nach sich selbst; alsdann wächst [...] die allgemeine Vollkommenheit und einer nicht verächtlichen Generation folgt immer eine noch bessere nach. ${ }^{125}$
\end{abstract}

Die Pluralform verweist auf die Kongruenz von individuellem und nationalem Charakter, während die Fernhaltung von „Verwirrung und Unruhe“ der zuvor zitierten Vermeidung der „Entfremdung“ entspricht. Anschließend macht Humboldt den entscheidenden Schritt in Richtung Menschheit: nur die Ausbildung jeweils spezifıscher nationaler Identitäten garantiere deren „Einheit im Ganzen“. Dies darf aber die implizite Differenzierung nicht verdecken, denn die zitierte „Tätigkeit der Kräfte“ verweist eindeutig auf die Produktivität des deutschen Idealismus und kontrastiert mit der postulierten Erschlaffung der französischen Kultur. Die ins Auge gefasste Abfolge der Generationen - ein Abgleiten der Differenzierung in die Hierarchisierung - kündigt ihrerseits den Paradigmenwechsel von „äußeren“ zu „inneren“ Handlungen an. Humboldts These wird noch deutlicher, wenn man sich an folgende Reflexion aus dem Fragment Über den Geist der Menschheit erinnert:

\footnotetext{
Wenn aber alles außer uns wankt, so ist allein noch in unserem Innern eine sichere Zuflucht offen, und seitdem in einem der bedeutendsten und cultiviertesten Teile der Erde eine wirkliche Umkehrung aller Verhältnisse stattgefunden hat, ist es immer zweifelhaft, wieviel sich in den übrigen davon erhalten wird ? zumal da jene Umkehrung in einem philosophischen Zeitalter als das einzig- Rechtmässige, als etwas absolut- und moralisch Nothwendiges vorgestellt wird. ${ }^{126}$
}

Die auf das „Innere“ konzentrierten mentalen Dispositionen der Deutschen lassen diese noch einmal als mustergültige Moderne erscheinen. Zugleich erweist sich diese Besonderheit laut Humboldt als effızientes Widerstandspotential gegen die durch die Revolution ausgelöste Krise Europas ${ }^{127}$ und verbindet sich mit der zweiten für den deutschen ,Nationalcharakter` spezifischen Besonderheit,

125. Humboldt, Über Hermann und Dorothea, S. 304.

126. Wilhelm von Humboldt, „Über den Geist der Menschheit“, in ders., Werke, Bd. I, S. 506.

127. Humboldt macht diese Widerstandskraft des „Inneren“ auch am Beispiel des Dramas deutlich: „Alles drängt sich hier dem Moment der Entscheidung entgegen, die Kraft des Geistes und des Charakters muß sich bis zur höchsten Anspannung sammeln, um die Macht des Schicksals zu überwinden, und sich ganz in sich selbst zurückziehn, um ihr nicht zu unterliegen." Humboldt, Brief vom 16. Oktober 1795, BW I, S. 181. 
nämlich der Offenheit für andere Kulturen ${ }^{128}$, um nun eine auf den Erfahrungen der Französischen Revolution aufbauende europäische Kultursynthese möglich zu machen. ${ }^{129}$

In seiner Auseinandersetzung mit der Revolution formuliert Humboldt zwar vorsichtige Zweifel an dem Universalismus der in Frankreich propagierten Werte ${ }^{130}$, konzentriert seine Kritik jedoch auf die revolutionäre Umwälzung als solche und beschäftigt sich zunehmend mit den positiven kulturellen Konsequenzen. Diese beruhen paradoxerweise auf dem in seinen Augen kontraproduktiven Verlauf der Französischen Revolution, was seine Ideen über Staatsverfassung belegen, die zugleich in einem anderen Licht erscheinen. Denn dort hatte er postuliert, die Vernunft alleine könne eine neue Verfassung nicht erfolgreich und dauerhaft durchsetzen, da die Kontingenz der Geschichte und das Beharrungsvermögen der Tradition sich ihr widersetzen ${ }^{131}$ und er hatte diesen Gedanken zu der These zugespitzt, ein Volk könne nie reif genug für eine allein auf Vernunft beruhende Umwälzung oder Revolution sein, da diese einseitig sei und kein „vereintes, und verhältnismäßiges Wirken aller Kräfte“" ${ }^{132}$ zulasse:

Bei jeglicher Umformung der Gegenwart muss auf den bisherigen Zustand ein neuer folgen. Nun aber bringt jede Lage, in welcher sich die Menschen befinden, jeder Gegenstand, der sie umgibt, eine bestimmte, feste Form in ihrem Innren hervor. Diese Form vermag nicht in jede andre selbstgewählte überzugehen, und man verfehlt zugleich seines Endzwecks und tötet die Kraft, wenn man ihr eine unpassende aufdringt. ${ }^{133}$

128. „Wir hingegen gewöhnen uns jetzt die Eigentümlichkeiten jeder Zeit und jeder Nation zu studieren, so viel wie möglich in dieselben einzugehen, und diese Kenntnis zum Mittelpunkt unsrer Beurteilung zu machen. Wenn jenes erstere Verfahren die andern Nationen in knechtischer Unterdrückung zurückhielt, und die Gemeinschaft mit ihnen erschwerte [...], so muss dieses letzere notwendig zugleich alle Kräfte wecken, die erwachten in Berührung bringen [...].“ Humboldt, „Das achtzehnte Jahrhundert“, S. 457.

129. „Der Gallomanie [...] folgte bald die Anglomanie nach, und diese beiden Nationen theilten dem übrigen Europa die Zwietracht mit, welche sie beide unter einander spaltet, bis auf und durch diese endlich die bessere und parteilosere Kultur durchzubrechen begann, der wir uns hetzt erfreuen, und welche den Deutschen in einem nicht wenig ehrwürdigen Lichte zeigt." Ibid., S. 501.

130. „[...] zumal da jene Umkehrung in einem philosophischen Zeitalter als das einzig- Rechtmässige, als etwas absolut- und moralisch Nothwendiges vorgestellt wird.“ Humboldt, „Über den Geist der Menschheit“, S. 506.

131. Humboldt, „Ideen über Staatsverfassung“ S. 34. Gerade deshalb wirft er Rousseau vor, dieser habe „das Reich der Ideen und das der Wirklichkeit“ nicht genügend von einander getrennt. Humboldt, Tagebücher, S. 572. Zum Verhältnis von Revolution und Reform bei Humboldt vgl. Christian Ferrié, „Humboldt contre la Révolution: l'impossible réforme“, in Agard / Lartillot, Le libéralisme de Humboldt, S. 89-119, insb. 97-98.

132. Humboldt, „Ideen über Staatsverfassung“, S. 34. Zur Verfestigung der Tradition durch die Revolution vgl. folgende Reflexion aus den Ideen zu einem Versuch: „Wer demnach die schwere Arbeit versuchen will, einen neuen Zustand der Dinge in den bisherigen kunstvoll zu verweben, der wird vor allem sie nie aus den Augen verlieren dürfen. Zuerst muss er daher die volle Wirkung der Gegenwart auf die Gemüter abwarten; wollte er hier zerschneiden, so könnte er zwar vielleicht die äußre Gestalt der Dinge, aber nie die Stimmung der Menschen umschaffen, und diese würde wiederum sich in alles Neue übertragen, was man gewaltsam ihr aufgedrungen hätte." Humboldt, Ideen zu einem Versuch, S. 195-196.

133. Ibid., S. 194. Vgl. auch die Betonung der notwendigen ,inneren“ Verankerung der Ideale: „Was im Menschen gedeihen soll, muss aus seinem Innren entspringen, nicht ihm von außen 
In diesem Sinne war für Humboldt die Revolution schon 1792 gescheitert; und vor allem hatte er schon damals das Theorem der nachlassenden Energie ins Spiel gebracht, das ihm während seines zweiten Pariser Aufenthalts zur zentralen Erklärung für den angeblichen kulturellen Niedergang Frankreichs wird. Kurz und gut, ein brutaler Umschlag in bürgerliche Freiheit schade deren dauerhafter Durchsetzung, wodurch zugleich in dem betroffenen Staat „jeder fernere Fortschritt“ ${ }^{134}$ der Aufklärung unmöglich werde. Ein solcher Misserfolg schließe aber positive Auswirkungen der Revolution in anderen Staaten bzw. Kulturen nicht aus:

Ob diese Staatsverfassung Fortgang haben wird? Der Analogie der Geschichte nach, Nein! Aber sie wird die Ideen aufs neue aufklären, aufs neue jede thätige Tugend anfachen, und so ihren Segen weit über Frankreichs Gränzen verbreiten. Sie wird dadurch den Gang aller menschlichen Begebenheiten bewähren, in denen das Gute nie an der Stelle wirkt, wo es geschieht, sondern in weiten Entfernungen der Räume oder der Zeiten, und in denen jene Stelle ihre wohlthätige Wirkung wieder von einer andren, gleich fernen, empfängt. ${ }^{135}$

Humboldts Blick ist in die Zukunft gerichtet und damit sind wir endgültig bei den Deutschen, ihren psychologischen Dispositionen und den daraus resultierenden welthistorischen Konsequenzen angelangt:

Denn das Wahre, sobald es - wäre es auch nur in einem Menschen - tief eindringende Wurzeln fasst, verbreitet immer, nur langsamer und geräuschloser, heilsame Folgen auf das wirkliche Leben; da hingegen das, was unmittelbar auf dasselbe übertragen wird, nicht selten bei der Übertragung selbst seine Gestalt verändert und nicht einmal auf die Ideen zurückwirkt. ${ }^{136}$

Die Semantik belegt bis zur Karikatur, dass Humboldt noch einmal Franzosen und Deutsche einander gegenüberstellt, um der Revolution den Rücken zuzukehren und die kommende kulturelle Synthese ins Auge zu fassen. Dies beweist auch sein Brief an Goethe kurz nach der Absendung der französischen Kurzfassung seines Essays über das Epos an Mme de Staël, in dem es heißt, diese Arbeit habe ihn sehr interessiert, da er gelernt habe, „welche Winkelzüge man machen muss, wenn man mit französischem Wind in eine deutsche Richtung segeln will." ${ }^{137}$

gegeben werden, und was ist ein Staat, als eine Summe menschlicher wirkender und leidender Kräfte? Humboldt, „Ideen über Staatsverfassung“, S. 36. Christian Helmreich erfasst Humboldts Gegenüberstellung von Revolution und Kontinuität zu Recht als Gegensatz von Außen und Innen. Wenn er jedoch schreibt, politische Revolutionen resultieren aus „inneren Revolutionen“ und die „Geschichte der Welt“ sei nur „die Widerspiegelung der Geschichte unserer Innerlichkeit“, dann verallgemeinert er all zu sehr und übersieht Humboldts Unterscheidung von Ideen $\dot{a}$ la française und Idealen im Sinne des deutschen Idealismus. Helmreich, „L'Histoire“, S. 82-84.

134. Humboldt, Ideen zu einem Versuch, S. 202.; vgl. Ferrié, „Humboldt contre la Révolution“, S. 113.

135. Humboldt, „Ideen über Staatsverfassung“, S. 40. Vgl. auch folgende gegen Gentz und Burke gerichtete Stellungnahme: „Denn die Wahrheiten der französischen Revolution bleiben ewig Wahrheiten, wenn auch 1200 Narren sie entweihen.“ Wilhelm von Humboldt, Brief an Brinkmann vom 9. November 1792, in ders., Briefe, Bd. 2, Juli 1791-Juni 1795, hrsg. und kommentiert von Philip Mattson, Berlin, de Gruyter 2015, S. 102.

136. Humboldt, Ideen zu einem Versuch, S. 193.

137. Humboldt, Brief an Goethe vom 30. Mai 1800, in Franz Thomas Bratranek (Hrsg.), Goethes Briefwechsel mit den Gebrüdern Humboldt, [1876], ND Bremen, Europäischer Literaturverlag, 2014, S. 161. Wie strategisch Humboldts Essay über Goethe geschrieben ist, beweist in diesem 
Die Denkbewegung bringt zunächst das zukunftsträchtige Verhältnis von Französischer Revolution und deutschem Idealismus zum Ausdruck. Frankreich sei erschöpft, habe aber durch seine politische die deutsche philosophische Revolution ausgelöst, welche als Widerstand gegen das ausschließlich Politische und als dessen intellektuelle Verarbeitung zugleich zu verstehen ist. Dieses Postulat entspricht den von Schiller in seinem zwischen 1797 und 1801 verfassten Fragment Deutsche Größe formulierten Thesen und dessen Tendenz, das Universelle vom Nationalen abzuleiten, das Universelle durch das Nationale zu erreichen. ${ }^{138}$ Schiller spricht an der entsprechenden Stelle seiner Argumentation unverblümt von der kommenden Herrschaft des deutschen Geistes:

Dem, der den Geist bildet, beherrscht,

Muss zuletzt die Herrschaft werden, denn

Endlich an dem Ziel der Zeit, wenn anders die

Welt einen Plan, wenn des Menschen

Leben irgend nur Bedeutung hat,

Endlich muss die Sitte und die Vernunft siegen,

Die rohe Gewalt der Form erliegen -

und das langsamste Volk wird alle

die schnellen flüchtigen einhohlen [sic]. ${ }^{139}$

Sicher, er denkt nur an die ,geistige“ Herrschaft der Deutschen, ${ }^{140}$ die dem „Plan“ der Geschichte entspreche. Trotzdem lässt er sich jedoch gehen, wenn er diese angebliche Rolle als Auserwähltheit durch den „Weltgeist“ ${ }^{141}$ bezeichnet. So weit geht Humboldt nicht, wenn er seine zentralen Thesen zur Rolle der Deutschen zusammenfasst:

Zusammenhang folgende Reflexion: „Aus dem Untergang und der Zerstörung sehen wir neues Leben, aus der Verwirrung der Völker das Glück und die fortschreitende Veredlung einer Familie hervorgehen." Humboldt, Über Hermann und Dorothea, S. 307.

138. Vgl. Georg Schmidt, „Friedrich Schillers Deutsche Größe und der nationale Universalismus”, in Werner Greiling / Hans-Werner Hahn (Hrsg.), Tradition und Umbruch. Geschichte zwischen Wissenschaft, Kultur und Politik, [FS Herbert Gottwald], Rudolstadt/Jena, Hain, 2002, S. 11-32. Zur Wirkungsgeschichte vgl. Dieter Borchmeyer, Was ist deutsch ? Variationen eines Themas von Schiller über Wagner zu Thomas Mann, Eichstätt, kath. Universität Eichstätt-Ingoldstadt, 2010.

139. Es wurde 1902 erstmals von Suphan veröffentlicht: Friedrich Schiller, Deutsche Grösse, in Schillers Werke, Nationalausgabe, Zweiter Band, Teil I, Gedichte, hrsg. von Norbert Oellers, Weimar, Böhlau, 1983, S. 433, Verse 1-14. Angesichts dieser Töne ist auch Manfred Fuhrmann $\mathrm{zu}$ widersprechen, wenn er schreibt, Schiller spreche bei seiner Absetzung von den Alten allgemein von den Modernen und denke nicht in nationalen Kategorien. Vgl. Fuhrmann, „Die ,Querelle des Anciens et des Modernes““, S. 56.

140. Zur politischen Instrumentalisierung des Textes vgl. J. H. Ulbricht, „Was ist heut“ des Deutschen Größe?", in Michael Knoche (Hrsg.), Das ,deutsche Buch ' in der Debatte über nationale Identität und kulturelles Erbe, Göttingen, Wallstein, 2006, S. 28-45.

141. Ibid., Vers 17. 
Und dir gab das Geschick, die Höhen und Tiefen der Menschheit

Eigner und besser zu schauen, höher und reicher die Kraft.

Denn die Sprache Teutoniens ist's, die, geschmeidiger Bildung,

Einst dir des ahnendes Geists Erstlingsgedanken erschließt;

Sie, die von eigenem Stamm entsprossen, und kräftig und edel

Näher des Griechen Flug rauschende Fittige schwingt.

Wenig wird noch erkannt das Volk, das still und bescheiden,

Aber tieferen Ernsts kühnere Bahnen sich bricht;

Doch sie kommt, die vergeltende Zeit, schon winkt sie nicht fern mehr,

Wo es dem Folgegeschlecht zeichnet den leuchtenden Pfad.

Nicht mit Waffen wird es, nicht kämpfen in blutigen Kriegen,

Sichrer herrschet durchs Wort, edler sein schaffender Geist.

Doch auch er verfällt am Ende einem übel aufstoßenden Revanchismus:

Das nur können die Eltern, nur das allein Dir gewähren,

Dass sie mit Deutschem Sinn sorgsam Dich nähren und früh,

Was sie besassen an Kraft, und was sie sich mühsam erstrebten,

Haben sie innig und treu Dir in die Seele gehaucht;

Geh' nur selbst es vollendend und zeige dem kommenden Enkel,

Dass Dich zum Weichling nicht zeugt' ein entartet Geschlecht. ${ }^{142}$

\section{Ausblick}

Die Deutschen erweisen sich für Humboldt als die exemplarischen Modernen, die die französische Herrschaft „äußerer Handlungen“ durch die ihnen eigenen „inneren Handlungen“ ablösen. Dies bedeutet zugleich, dass sie nicht nur die Nachfolge der Griechen antreten, sondern diese auch übertreffen, was allerdings dem allgemeinen kulturellen Fortschritt der Modernen geschuldet ist. ${ }^{143}$ Die Franzosen ihrerseits können vielleicht eines Tages „eine große Tiefe des Geistes“ entwickeln und dann, endlich, „wohlthätig für Europa“144 werden, was einer „Art der Wiedergeburt“ ${ }^{145}$ gleichkäme, ${ }^{146}$ die allerdings eine nachdrückliche Aufnahme des deutschen Idealismus voraussetzt und

142. Wilhelm von Humboldt, „An den erwarteten Sohn“, in ders., Gesammelte Schriften, Bd. IX, S. 13-14. Der Hinweis auf diesen selten zitierten Text bei Aira Kemiläinen, Auffassungen über die Sendung des deutschen Volkes um die Wende des 18. und 19. Jahrhunderts, Helsinki, Suomalainen Tiedeakatemia, 1956, die seltsamerweise keinen direkten Bezug zu Schiller herstellt (S. 124 und, zu Schiller, S. 142-143).

143. „Denn wenngleich die Alten mehr sinnliche Schönheit der Sprache besitzen, so hat unsere Sprache - vermöge des Fortrückens der Menschheit überhaupt - eine feinere intellektuelle Ausbildung und eine den Menschen tiefer und innerlicher bewegende Kraft." Humboldt, Brief an Schiller Anfang September 1800, BW II, S. 209-210.

144. Humboldt, Brief an Jacobi vom 26. Oktober 1798, S. 70.

145. Ibid.

146. Auch dieser Gedankengang findet sich schon in den Reflexionen zur französischen Verfassung vorformuliert, in denen es heisst: „In jeder Epoche desselben [des Menschenlebens] ist Eine Art des Daseins Hauptfigur in dem Gemälde, indess alle übrigen ihr, als Nebenfiguren, dienen. In einer andren Epoche wird sie zur Nebenfigur, und eine andre von jenen tritt auf den Vordergrund.“ Humboldt, „Ideen über Staatsverfassung“, S. 41. Zu Humboldts Historismus vgl. Georg G. Iggers, Deutsche Geschichtswissenschaft, Wien, Böhlau, 1997, S. 62-85. 
daher unwahrscheinlich ist. Was Humboldts Briefe betrifft, so sind sie dank der Orientierung an den Vorgaben der Horen ästhetisch kohärente Fragmente, Bausteine zu dem kulturgeschichtlichen Aufsatz über Frankreich, den er nie schreiben wird. 